\title{
Nanostructured lipid carriers loaded with resveratrol modulate human dendritic cells
}

\author{
This article was published in the following Dove Press journal: \\ International Journal of Nanomedicine \\ 28 July 2016 \\ Number of times this article has been viewed
}

\author{
João P Barbosa ${ }^{1-3, *}$ \\ Ana R Neves ${ }^{3, *}$ \\ Andreia M Silva ${ }^{1,2,4}$ \\ Mário A Barbosa ${ }^{1,2,4}$ \\ M Salette Reis ${ }^{3}$ \\ Susana G Santos ${ }^{1,2}$ \\ 'Instituto de Investigação e Inovação \\ em Saúde, Universidade do Porto, \\ Portugal; ${ }^{2}$ INEB - Instituto de \\ Engenharia Biomédica, Universidade \\ do Porto, Portugal; ${ }^{3} \mathrm{UCIBIO}$, \\ REQUIMTE, Chemical Sciences \\ Department, Faculty of Pharmacy, \\ University of Porto, Portugal; \\ ${ }^{4}$ Instituto de Ciências Biomédicas \\ Abel Salazar, Universidade do Porto, \\ Porto, Portugal \\ *These authors contributed equally \\ to this work
}

\begin{abstract}
Dendritic cells (DCs) are promising targets for drug delivery, as they can induce immunity or tolerance. The current study aims to examine the potential of using nanostructured lipid carriers (NLC) as delivery systems for human DC by evaluating nanoparticle internalization, cell labeling, and drug activity. NLC were formulated incorporating the fluorochrome fluorescein isothiocyanate (FITC-NLC) or the natural anti-inflammatory molecule resveratrol (rsv-NLC). Primary human DCs were differentiated from peripheral blood monocytes, and the innovative imaging flow cytometry technique was used to examine FITC-NLC internalization. The capacity of rsv-NLC to inhibit DC activation in response to proinflammatory cytokine tumor necrosis factor- $\alpha$ (TNF- $\alpha$ ) was investigated by conventional flow cytometry. A combination of imaging and conventional flow cytometry was used to assess NLC cytotoxicity. The results obtained indicate that both NLC formulations were stable over time, with mean diameter $<200 \mathrm{~nm}$ and highly negative zeta potential (about $-30 \mathrm{mV}$ ). When DCs were placed in contact with NLC, imaging flow cytometry clearly showed that DCs efficiently internalized FITC-NLC, with nearly $100 \%$ of cells internalizing nanoparticles upon 1 hour of incubation. Both immature and mature DCs internalized NLC to high and comparable levels, and without cytotoxicity. Stimulating DC with TNF- $\alpha$ in the presence of rsv-NLC revealed that, using these nanoparticles, very small concentrations of rsv were sufficient to significantly decrease surface expression of activation marker CD83 $(5 \mu \mathrm{M})$ and major histocompatibility complex-class II molecule human leukocyte antigen - antigen D related $(10 \mu \mathrm{M})$, both upregulated in response to TNF- $\alpha$ stimulation. Rsv-NLC were compared with free rsv; at $5 \mu \mathrm{M}$, rsv-NLC were able to inhibit nuclear factor $\kappa$ beta phosphorylation and significantly decrease the level of interleukin-12/23, both upregulated in response to TNF- $\alpha$, while $10 \mu \mathrm{M}$ free rsv were needed to promote a similar effect. Taken together, the results presented show that NLC are suitable carriers of fluorescent labels or bioactive molecules for human DCs, leading to inflammation modulation.
\end{abstract}

Keywords: immunomodulation, dendritic cell, solid lipid nanoparticle, imaging flow cytometry, resveratrol, TNF- $\alpha$

\section{Introduction}

Dendritic cells (DCs) are the most potent professional antigen-presenting cells, and so are key players that link the innate and adaptive immune responses. This role has placed them as the most ambitious targets for immunomodulatory therapies, both for promoting immunity, ${ }^{1}$ in fighting cancer, or infection and for promoting tolerance, ${ }^{2}$ as in autoimmunity. In addition, DCs are responsible for immune surveillance and move from the periphery to secondary lymphoid organs, where immune responses can be triggered. Thus, DC labeling could be an important tool in evaluating the efficacy of immunotherapy. ${ }^{1}$

The natural antioxidant and anti-inflammatory compound resveratrol (rsv) is found in a variety of plants and fruits that constitute our normal diet, such as grapes and also
Correspondence: Susana G Santos Instituto de Investigação e Inovação em Saúde, Rua Alfredo Allen 208, 4200-I 35 Porto, Portugal

Tel +35I 220408800 ext 6075 Email susana.santos@ineb.up.pt
International Journal of Nanomedicine 20|6: | | 350|-35|6

Dovepress f $y$ in $\mathbf{0}$

http://dx.doi.org/10.21477/IN.S108694
C. $\odot 2016$ Barbosa et al. This work is published and licensed by Dove Medical Press Limited. The full terms of this license are available at https://www.dovepress. com/terms.php hereby accept the Terms. Non-commercial uses of the work are permitted without anny further permisision from Dove Medical Press limited, provided the work is properly attributed. For permission or commercial use of this work, please see paragraphs 4.2 and 5 of our Terms (https://www.dovepress. com/terms.php). 
in wine, where it was believed to be the reason behind the so-called French paradox. ${ }^{3}$ Rsv has strong anti-inflammatory properties and is currently being investigated for the treatment of a wide variety of disorders, from cancer to autoimmunity, ${ }^{3}$ with over 100 clinical trials registered (ClinicalTrials.gov). rsv has been shown to act as a potent anti-inflammatory agent in cells ${ }^{4-8}$ and animal models, including a rabbit model of arthritis. ${ }^{9}$ Interestingly, rsv has been shown to be able to inhibit DC maturation in response to lipopolysaccharide. ${ }^{10,11}$ Moreover, our recent work shows that it can modulate DC response to inflammatory cytokine tumor necrosis factor- $\alpha$ (TNF- $\alpha) .{ }^{12}$ The mechanisms of rsv action on DC have been reported by us and others to involve inhibition of nuclear factor $\kappa$ beta (NF-אB) activation, ${ }^{11-15}$ which is known to lead to proinflammatory cytokine production. However, these studies use free rsv, which has low water solubility and bioavailability, ${ }^{3}$ forcing the use of high doses, which may result in toxicity ${ }^{16}$ and dedifferentiation, particularly reported for DCs. ${ }^{11}$

Rsv encapsulation in different nanodelivery systems has been the subject of intense investigation in recent years., ${ }^{3,17}$ Lipid nanoparticles present a lot of advantages as systems for drug delivery, namely, for lipophilic compounds, since they are generally composed of biodegradable and biocompatible excipients tolerated by human body and have a high physical stability. ${ }^{18}$ The preparation of lipid nanoparticles is a water-based technology, avoiding organic solvents that could be a source of toxicity. Moreover, these nanosystems are economically more affordable and easier to scale-up than other nanocarriers. ${ }^{19}$ Previous work has demonstrated that both solid lipid nanoparticles and nanostructured lipid carriers (NLC) are suitable vehicles for rsv encapsulation, ${ }^{20}$ but NLC were found to perform better. ${ }^{21}$ In fact, NLC are composed of a mixture of both solid and liquid lipids at room temperature (RT), creating an imperfect matrix with a larger number of cavities, ${ }^{22}$ which allows a better accommodation of the encapsulated compound and prevents early crystallization and subsequent release at storage. ${ }^{20}$

The use of lipid nanoparticles has been previously tested for delivery of other compounds to $\mathrm{DC},{ }^{23,24}$ but the use of nanoparticles to deliver rsv to DC has not been reported previously. Therefore, the current study aimed to test the suitability of NLC as drug carriers for DC, for therapeutic or labeling purposes, and the capacity of rsv encapsulated in NLC to modulate DC phenotype in a proinflammatory environment.

\section{Materials and methods}

\section{Ethics statement}

Human biological samples used in this work and all the procedures followed to obtain them were according to the principles of the Declaration of Helsinki. Monocytes were isolated from surplus buffy coats from healthy blood donors, kindly donated by Serviço de Imunohemoterapia Centro Hospitalar de São João (CHSJ, Porto, Portugal), as part of an agreement with our institute. Blood donors gave informed written consent for the by products of their blood collections to be used for research purposes, as approved by the Ethics Committee for Health from CHSJ. No information on age, sex, or any identifying element was provided to the researchers, so all samples were analyzed anonymously.

\section{NLC production}

NLC were produced as previously described..$^{20}$ Briefly, the lipid components of the future emulsion (Table S1) were weighed, and both lipid and aqueous phases were heated separately, up 5-10 degrees above the melting temperature of the lipids, causing complete melting of this phase. Subsequently, both phases were mixed to promote formation of oil/water emulsion. The mixture was then submitted to high-speed stirring $(12,000 \mathrm{rpm})$ in an Ultra-Turrax T25 (Janke and Kunkel IKA-Labortechnik, Staufen, Germany), followed by sonication using a Vibra-Cell ${ }^{\mathrm{TM}}$ CV18 (Sonics \& Materials, Inc., Newtown, CT, USA) in order to form nanoscale particles. Cooling to RT allowed lipid crystallization and formation of lipid nanoparticles. This method was used to synthesize non-loaded NLC, fluorescein isothiocyanate-loaded NLC (FITC-NLC), and rsv-loaded NLC (rsv-NLC), where FITC or rsv were added to the lipid phase during NLC production. Nanoformulations were then stored at RT and protected from light in order to perform a stability control study.

\section{NLC characterization}

Average diameter of the nanoparticles and polydispersity index (Pdi) were measured using dynamic light scattering, and zeta potential (ZP) determined using electrophoretic light scattering - both measured using Zeta PALS Zeta Potential Analyser (Brookhaven Instruments Corporation, Holtsville, NY, USA). Fluorescence of NLC formulations was measured using a luminescence spectrometer LS50B (PerkinElmer, Santa Clara, CA, USA). The entrapment efficiency (EE) of rsv was determined by an indirect method described elsewhere, ${ }^{20}$ assessing the amount of free rsv that was still present in the aqueous phase using a V-660 spectrophotometer (Jasco, Easton, MD, USA) at 200-600 nm, with a maximum absorption at $306 \mathrm{~nm}$. Size, Pdi, ZP, and EE was measured monthly, up to 3 months, and measurements were expressed as mean \pm standard deviation, with $n \geq 3$. 


\section{Electron microscopy}

NLC morphology was characterized by cryo-scanning electron microscopy (cryo-SEM) and transmission electron microscopy (TEM). For cryo-SEM analysis, NLC dispersions were cooled in a liquid nitrogen slush $\left(-210^{\circ} \mathrm{C}\right)$ on a grid, fractured, sublimated ( 4 minutes, $-90^{\circ} \mathrm{C}$ ) to reveal greater detail, and coated with a gold-palladium alloy. Finally, samples were observed at $-150^{\circ} \mathrm{C}$ under JSM-6301F (Jeol, Tokyo, Japan) and INCA Energy 350 (Oxford Instruments, Abingdon, UK) scanning electron microscopes and recorded using an Alto 2500 camera (Gatan, Inc., Pleasanton, CA, USA). For TEM analysis, samples were mounted on 300 mesh formvar copper grids, counterstained with uranyl acetate, and examined using a JEM 1400 transmission electron microscope (Jeol) and recorded using a Orius SC 1000 charge-coupled device camera (Gatan, Inc., Warrendale, PA, USA).

\section{Primary DC cultures}

Monocyte isolation was performed as described. ${ }^{25}$ Briefly, peripheral blood mononuclear cells were collected from centrifuged buffy coat and incubated with RosetteSep human monocyte enrichment kit (StemCell Technologies SARL, Grenoble, France), according to manufacturer's instructions. The mixture was diluted with phosphate buffered saline (PBS) supplemented with heat-inactivated fetal bovine serum (FBS) (Lonza, Basel, Switzerland), layered over Histopaque ${ }^{\circledR}-1077$ (Sigma Aldrich GmbH, Steinheim, Germany), and centrifuged as before. The enriched monocyte layer was collected and washed with PBS. Population purity was evaluated and over $70 \%$ of the cells were found to be CD14 positive. ${ }^{25}$ Cells were plated at $1 \times 10^{6}$ cells $/ \mathrm{mL}$ in complete culture media (Roswell Park Memorial Institute$1640+$ Glutamax, with $10 \%$ heat-inactivated FBS and $1 \%$ penicillin G-streptomycin [Invitrogen, Paisley, UK]), further supplemented with $50 \mathrm{ng} / \mathrm{mL}$ interleukin (IL)-4 and granulocyte-macrophage colony-stimulating factor (Immunotools, Friesoythe, Germany). Monocyte-derived DCs were differentiated for 4-5 days to obtain immature DCs. ${ }^{26,27}$

\section{FITC-NLC internalization and imaging flow cytometry}

Before usage, a dilution (1:180) from nonloaded or FITCloaded NLC formulations, described in Table S1 was made (final volume: $1,800 \mu \mathrm{L}$ ). This solution was washed using Amicon ${ }^{\circledR}$ Ultra-4 centrifugal filter units (Merck Millipore ${ }^{\mathrm{TM}}$, Billerica, MA, USA), discarding the product of filtration. The suspension was then sterilized by filtration with $0.22 \mu \mathrm{m}$ filters (Merck Millipore).
Increasing volumes of FITC-NLC suspension were then added to unstimulated or TNF- $\alpha$ stimulated (100 ng/ $\mathrm{mL})$ DCs to evaluate NLC internalization. The DCs were incubated with NLC for 1 hour and then harvested, washed with PBS, and stained for DC lineage marker CD1a-PE (Immunotools) in staining buffer (PBS, 2\% FBS, 0.01\% azide) for 30 minutes on ice. Cells were then fixed using paraformaldehyde $4 \%$ and washed with PBS. Samples were filtered (40 mm mesh) and acquired using an ImageStream ${ }^{\mathrm{X}}$ (Billerica, Massachusetts, USA), imaging flow cytometer with INSPIRE software (Amnis, EMD Merck Millipore), at the Bioimaging Center for Biomaterials and Regenerative Therapies (b.IMAGE). Data were analyzed with IDEAS software (Amnis, EMD Merck Millipore). For each sample, 10,000 cells were acquired, and $>5,000$ single focused cells were analyzed for NLC internalization, using the "Internalization" wizard (Figure S1).

\section{DC stimulation and flow cytometry}

Immature DCs were treated with different amounts of NLC, diluted from formulations described in Table $\mathrm{S} 1$, in presence or absence of $100 \mathrm{ng} / \mathrm{mL}$ of TNF- $\alpha$ (Immunotools), and allowed to mature for 72 hours, before being harvested. Flow cytometry was performed as previously described ${ }^{25}$ Briefly, cells were washed and labeled (for 45 minutes at $4{ }^{\circ} \mathrm{C}$ in the dark) in staining buffer with combinations of the following antihuman antibodies: CD1a, CD11c, CD86-FITC, CD40FITC, HLA-ABC-PE, human leukocyte antigen - antigen D related (HLA-DR-PE) (all from Immunotools), CD83-FITC (AbDSerotec, Kidlington, UK). Isotype and fluorochromematched control antibodies were used to define background staining. For cell viability, Annexin V-FITC staining was performed for 15 minutes, followed by propidium iodide (PI; BD Biosciences, San Jose, CA, USA) staining, just prior to acquisition. For each sample, 10,000 cells were acquired, gated according to forward and side scatter parameters. Samples were analyzed using a FACS Calibur flow cytometer with Cell Quest software, or FACS Canto II flow cytometer, with Diva software (all from BD Biosciences, Franklin Lakes, New Jersey, USA). Results were analyzed using FlowJo software (TreeStar, Inc., Ashland, OR, USA). The mean fluorescence intensity for each sample was calculated subtracting the mean fluorescence intensity of the respective isotype control.

\section{Western blotting}

DCs were stimulated with TNF- $\alpha$ in presence or absence of rsv-NLC $5 \mu \mathrm{M}$, or $10 \mu \mathrm{M}$ of free rsv, for the indicated times. Cells were then harvested and washed twice with cold PBS, before cell lysis in the presence of protease and phosphatase 
inhibitors. Lysates were centrifuged (14,000 rpm, 10 minutes, $4^{\circ} \mathrm{C}$ ) and protein was quantified using the $D C^{\mathrm{TM}}$ Protein Assay (Bio-Rad Laboratories Inc., Hercules, CA, USA). The same amount of protein per sample was resolved by sodium dodecyl sulfate polyacrylamide gel electrophoresis in reducing conditions, and transferred to nitrocellulose membranes, which were blocked with 5\% nonfat dry milk in PBS-T (PBS with 0.1\% Tween-20). Membranes were then probed with either anti-phospho-NF-кB p65 (Ser536) or anti-p65. Appropriate secondary antibodies conjugated to streptavidinhorseradish peroxidase were used for signal detection, upon membrane incubation with chemiluminescent substrate (ECL, GE Healthcare Chalfont St Giles, Buckinghamshire, UK) and exposure to X-ray films.

\section{Cytokine secretion}

Supernatants of DC cultures stimulated as indicated were collected and centrifuged $\left(14,000 \mathrm{rpm}, 5\right.$ minutes, $\left.4^{\circ} \mathrm{C}\right)$, before being assayed for IL-12/23 (p40 subunit), or IL-10 levels, by enzyme-linked immunosorbent assay (Legend Max Human ELISA kits; BioLegend, San Diego, CA, USA) according to manufacturer's protocol. Sample concentrations $(\mathrm{pg} / \mathrm{mL})$ were determined from mean absorbance values for each set of samples and compared to a standard calibration curve. The amount of secreted cytokine was normalized for the total amount of protein in culture supernatants, calculated using the $D C^{\text {TM }}$ Protein Assay (as earlier), and is presented as pg of cytokine per milligram of total protein.

\section{Statistical analysis}

Statistical analysis of NLC size, Pdi, ZP, and EE characterization was performed using SPSS software (v 18.0; IBM, Armonk, NY, USA). Otherwise, statistical analysis was performed using Prism5 software, vs5.0a (GraphPad Software, Inc., La Jolla, CA, USA). Gaussian distribution was tested with Kolmogorov-Smirnov normality test. For parametric sample sets, one-way analysis of variance, or repeated measures analysis of variance, followed by Bonferroni, Tukey and Dunnett post hoc tests were used for multiple comparisons. For nonparametric samples, Friedman test followed by Dunn's uncorrected multiple comparison test was applied. A value of $P<0.05$ was considered statistically significant.

\section{Results}

\section{NLC production and characterization}

NLC were produced by combining high shear homogenization with ultrasounds in order to produce particles in the nanometer range. The results obtained are depicted in
Figure 1 and show that both FITC- and rsv-loaded formulations have similar size (below $200 \mathrm{~nm}$ ), Pdi indicative of a monodisperse distribution (between 0.1 and 0.2 ), and a highly negative ZP (up to $-30 \mathrm{mV}$ ), suggesting good stability in solution. No significant changes were observed for 3 months after synthesis, when NLC were stored at RT and protected from light (Figure 1).

Concerning morphology, cryo-SEM and TEM images of NLC are depicted in Figure 2A and B, respectively. Both techniques allowed to conclude that NLC were almost spherical and uniform in shape with smooth surfaces. The mean diameter did not exceed $200 \mathrm{~nm}$ and there was no visible aggregation of particles. Moreover, the addition of FITC or rsv did not modify the NLC morphology.

The formulations obtained were found to have an EE value higher than $80 \%$ and no decreases were observed in rsv incorporation over time, further demonstrating the great stability of these formulations and the capacity of nanoparticles to retain the compound inside after 3 months of storage (Figure S2). This great stability is probably related to the lipid composition of nanoparticles, since NLC present a disordered lipid matrix conferred by the presence of the liquid lipid. In a recent study, we performed differential scanning calorimetry analysis, where the evaluation of the lipid order parameters of lipid nanoparticles provided important information on the physical stability of nanoparticles. ${ }^{20}$ The phase transition temperature, melting enthalpy, and degree of crystallinity of NLC indicate that the liquid lipid miglyol-812 reduces particle crystallinity and confers higher physical stability due to the presence of lattice defects in the lipid core, which possibly allows a better accommodation of rsv or FITC fluorophore, which may avoid the premature release of the compound or the loss of fluorescence signal.

\section{NLC are internalized by naive and stimulated DCs}

In order to study nanoparticle internalization by DCs, the fluorochrome FITC was loaded into NLC (FITC-NLC). Two different FITC amounts were used to determine specific NLC detection ( 2 and $5 \mathrm{mg}$ of FITC). Upon internalization, DCs were analyzed by imaging flow cytometry, using ImageStream ${ }^{\mathrm{X}}$. The workflow used for the IDEAS' "Internalization wizard" is illustrated in Figure S1. Results illustrated in Figure 3A show that FITC-containing NLC are identified specifically, as cells that were incubated with NLC loaded with $0 \mathrm{mg}$ of FITC showed no fluorescence. Encapsulating FITC beyond $2 \mathrm{mg}$ did not provide higher detectable fluorescence inside cells (Figure 3B). Moreover, by combining the FITC-NLC with 

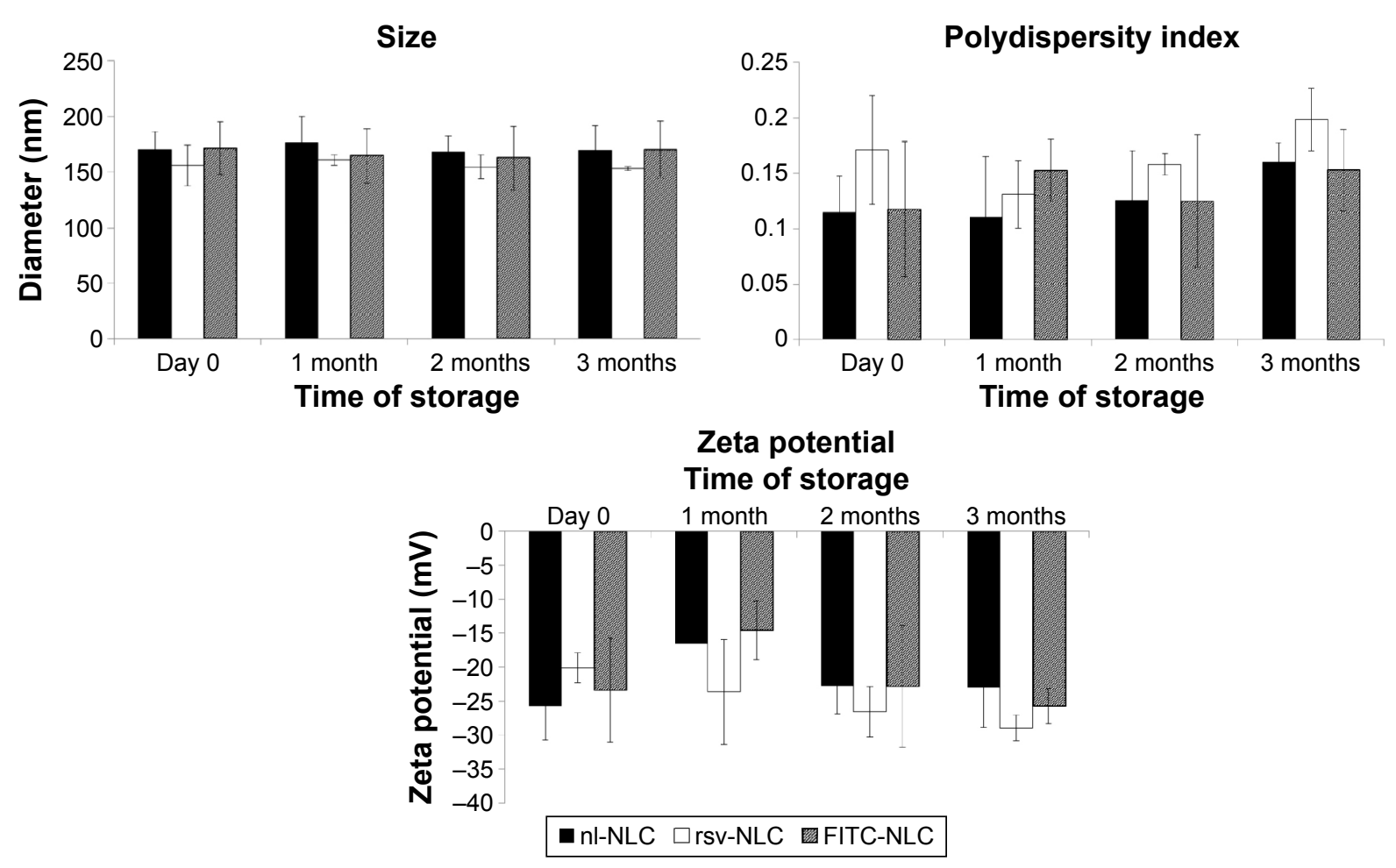

Figure I NLC characterization and stability.

Notes: NLC formulations nonloaded (nl-NLC), loaded with rsv molecules (rsv-NLC), and loaded with FITC fluorochrome (FITC-NLC) were analyzed for their size (top left), polydispersity index (top right), and zeta potential (bottom) by dynamic and electrophoretic light scattering when they were produced (day 0 ) and along time in storage. Values represent the mean $\pm S D(n \geq 3)$, for each parameter. No statistically significant changes were observed when compared to day 0 ( $P>0.05)$.

Abbreviations: FITC, fluorescein isothiocyanate; NLC, nanostructured lipid carriers; rsv, resveratrol; SD, standard deviation.

surface staining for the DC lineage marker CD1a, our results clearly show that NLC are internalized by DCs (Figure 3).

To determine the amount of NLC necessary for internalization by the vast majority of naive or activated DCs, increasing volumes of FITC-NLC $(25-200 \mu \mathrm{L})$ were added to the cell cultures, creating a series of nanoparticle concentrations. Results obtained are illustrated in Figure 4A,

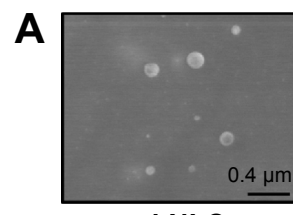

nl-NLC

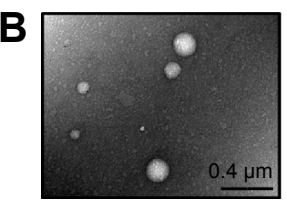

nl-NLC

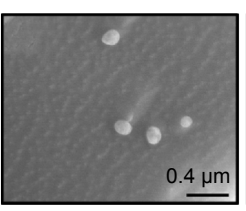

FITC-NLC

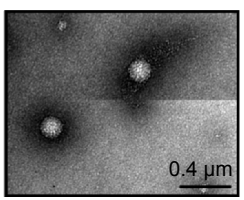

FITC-NLC

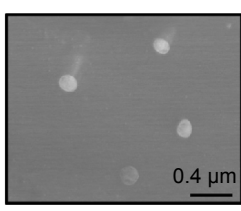

rsv-NLC

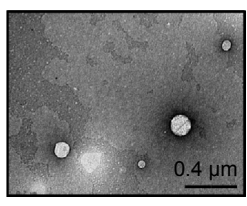

rsv-NLC
Figure 2 NLC morphology.

Notes: NLC formulations nonloaded (nl-NLC), loaded with FITC (FITC-NLC), and loaded with rsv (rsv-NLC) were analyzed by microscopy. (A) Cryo-scanning electron microscopy and (B) transmission electron microscopy representative images. Magnification: $\times 40,000$.

Abbreviations: FITC, fluorescein isothiocyanate; NLC, nanostructured lipid carriers; rsv, resveratrol. and reveal dose-dependent increase in NLC internalization. When results were quantified across different donors, close to $100 \%$ of the cells contained internalized NLC when $200 \mu \mathrm{L}$ of nanoparticles were used. As expected, DC stimulation with TNF- $\alpha$ results in lower NLC internalization; however, on quantification, differences between naive and activated cells were not statistically significant (Figure 4B). The $200 \mu \mathrm{L}$ of NLC were then used to investigate if stimulation of DCs would lead to a progressive loss of capacity to internalize NLC. Results in Figure 4C and D show that although there is decrease in uptake, even DCs stimulated 1 day before adding NLC, corresponding to early mature DCs, are still able to internalize NLC to comparable levels as naive cells.

\section{NLC are noncytotoxic for DC}

To determine if NLC had cytotoxic effects on DCs, cell morphology and induction of apoptosis (Annexin V/PI staining) were evaluated. Results illustrated in Figure 5A show no changes in cell morphology or granularity even when the highest concentration of FITC-NLC was used.

Moreover, when DCs were incubated with the same amounts of NLC as used for rsv experiments, and cells were then stained for apoptosis markers Annexin V/PI, no 

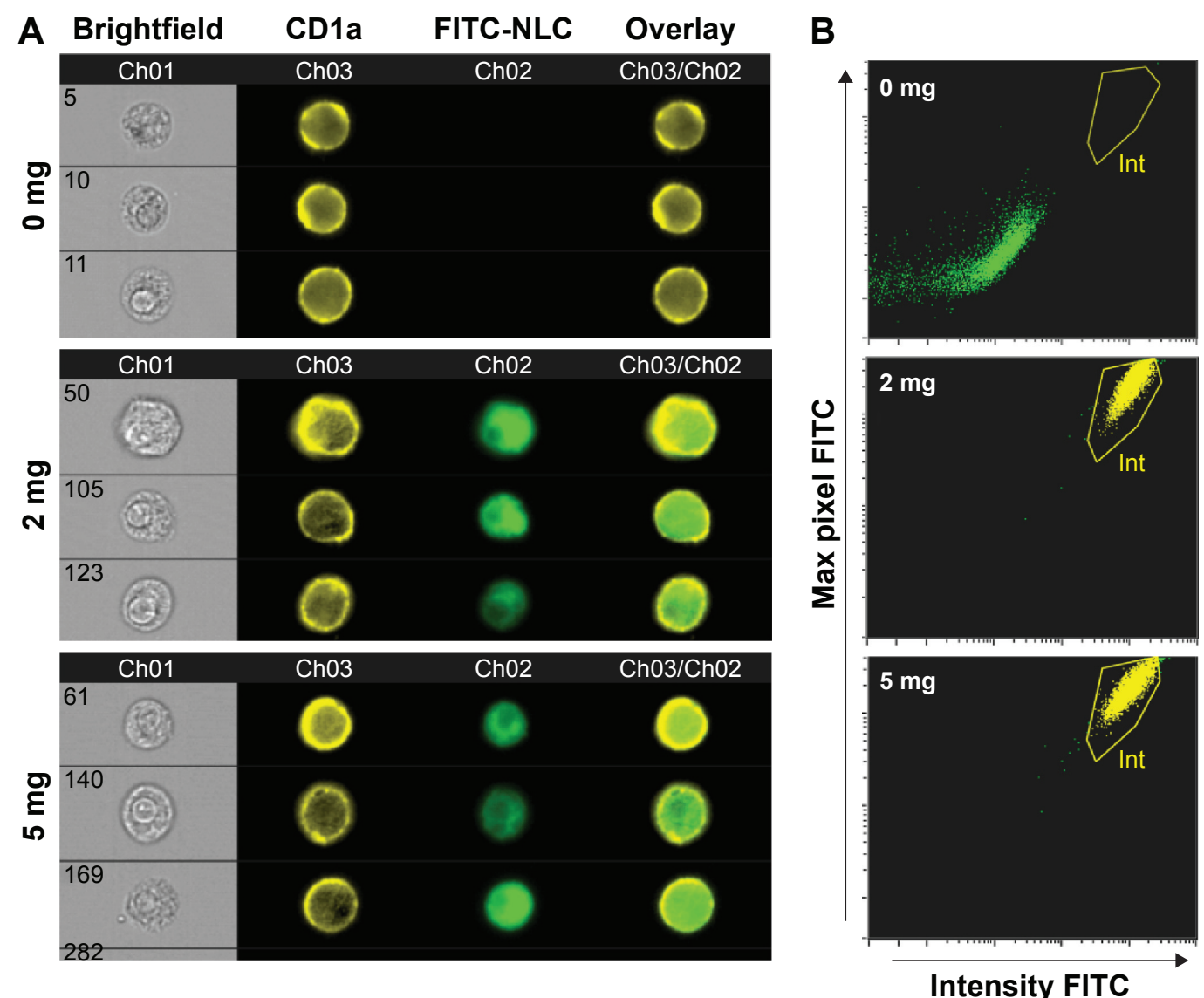

Figure 3 NLC internalization by DC, analyzed by imaging flow cytometry (ImageStream $\times$ ).

Notes: DCs were differentiated for 7 days before incubation with nonloaded $(0 \mathrm{mg})$ or FITC-NLC ( 2 and 5 mg) for I hour. Cells were then harvested and surface stained for the lineage marker CDIa before analysis by ImageStream ${ }^{\times}$. (A) Representative images of CDIa positive (yellow) DCs incubated with NLC loaded with 0 mg (top), $2 \mathrm{mg}$ (middle), or $5 \mathrm{mg}$ (bottom) of FITC (green), showing no detectable fluorescence for $0 \mathrm{mg}$, and evident intracellular green fluorescence for cells incubated with FITCNLC (2 or $5 \mathrm{mg}$ ). (B) Plots of the data illustrated in A for all the analyzed cells. The yellow regions represent the defined gate, corresponding to cells showing FITC-NLC internalization. Top graph, DCs incubated with NLC $0 \mathrm{mg}$ of FITC show FITC intensity values out of the internalization gate defined, while samples where FITC-loaded NLC were used, both for $2 \mathrm{mg}$ (middle) and $5 \mathrm{mg}$ (bottom) of FITC, fall inside the gate.

Abbreviations: DC, dendritic cell; FITC, fluorescein isothiocyanate; NLC, nanostructured lipid carriers; Int, internalized.

increase in single- or double-positive cells was observed (Figure 5B).

\section{Resveratrol encapsulated in NLC is bioactive and able to inhibit DC activation}

Having determined that NLC were internalized by DCs without cytotoxicity, we investigated their effectiveness as drug carriers. The anti-inflammatory molecule rsv was encapsulated into NLC, producing rsv-NLC and their effect on DC activation, in response to TNF- $\alpha$, was evaluated. Results in Figure 6A show a representative flow cytometric profile of DC stimulation with TNF- $\alpha$ and the consequences of rsv-NLC presence for the activation phenotype. Importantly, for all the conditions tested, the percentage of cells expressing the lineage markers $\mathrm{CD} 1 \mathrm{a}$ and $\mathrm{CD} 11 \mathrm{c}$ was maintained, indicating no dedifferentiation (Figure 6A).
On the contrary, the activation marker CD83 is represented together with lineage marker CD1a, while the costimulatory molecule CD86 and the major histocompatibility complex-II molecule HLA-DR are plotted against lineage marker CD11c. When results were quantified among different individuals (Figure 6B), it became clear that DCs significantly increase their cell surface expression of CD83, CD86, and HLA-DR in response to TNF- $\alpha$ stimulation, as expected. Moreover, the presence of rsv-NLC led to decreased DC activation, with a dose-dependent reduction of CD83 expression, which becomes statistically significant for rsv-NLC concentrations above $5 \mu \mathrm{M}$. In addition, HLA-DR was significantly reduced for rsv-NLC concentrations of $10 \mu \mathrm{M}$. Expression of CD86 was shown to be variable along the different rsv concentrations and for different donors, with a tendency to decrease, but that is not statistically significant. 
A FITC-NLC:

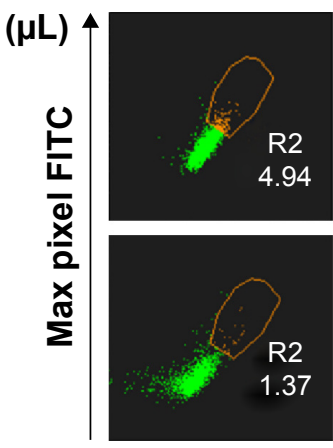

B

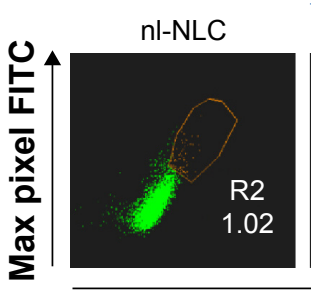

50
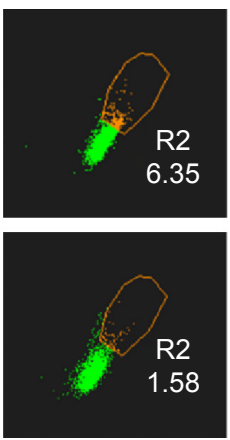

R2

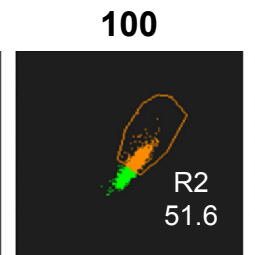

200
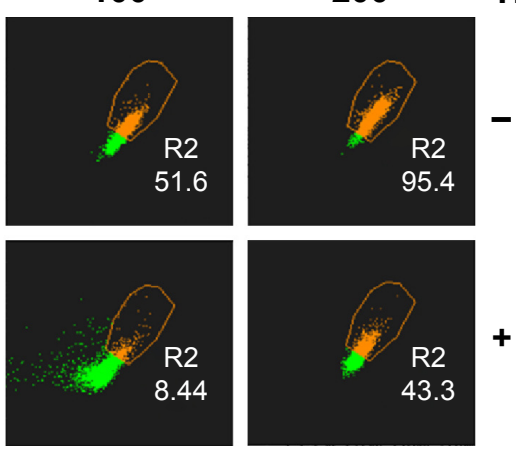

FITC-NLC

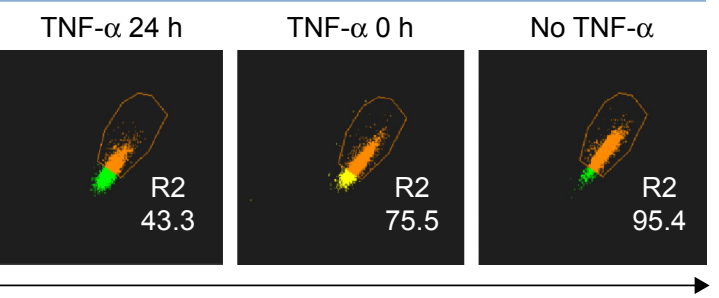

Intensity FITC

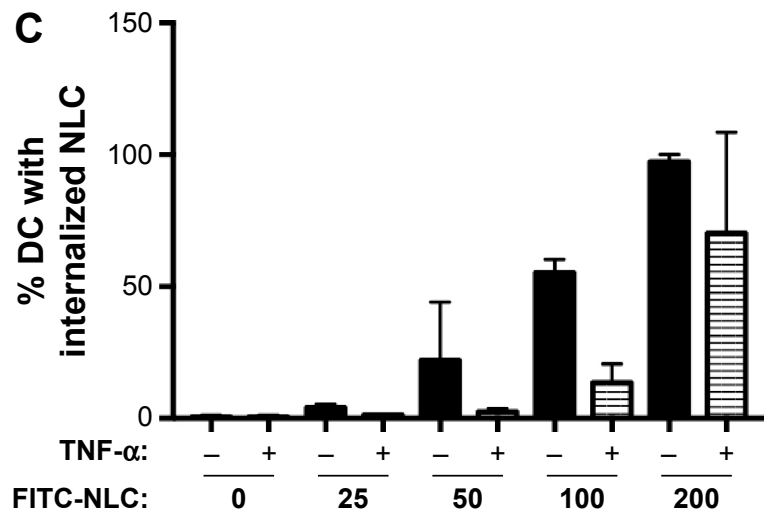

$(\mu \mathrm{L})$

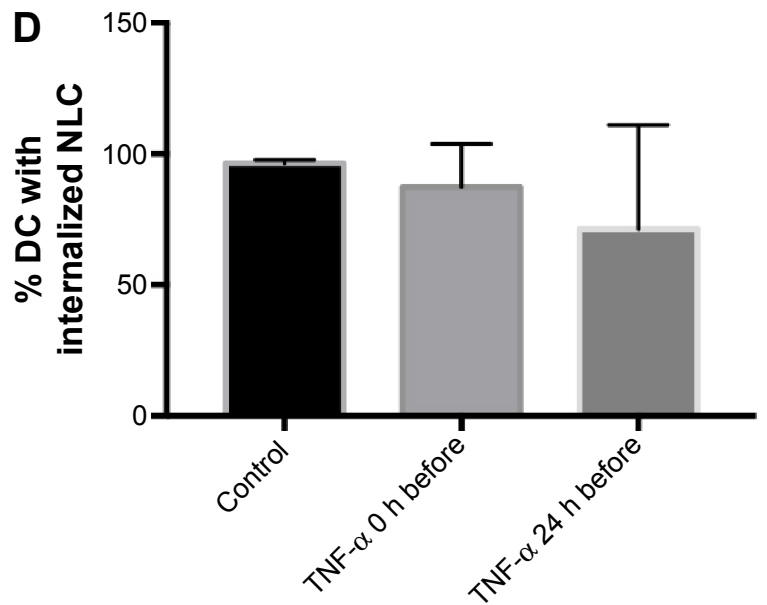

Figure 4 Immature and mature DCs internalize NLC.

Notes: (A) Differentiated DCs were left unstimulated or stimulated with TNF- $\alpha$ for 24 hours, then the cells were incubated with increasing volumes of FITC-NLC for I hour, before being harvested and surface stained for CDIa before analysis by ImageStream ${ }^{\times}$. (B) DCs were incubated with $200 \mu L$ of FITC-NLC unstimulated or stimulated with TNF- $\alpha$ simultaneously or 24 hours prior to incubation with NLC. Values represent percentage of cells in gate R2, with detectable FITC-NLC internalization. (C and D) Quantification of the results illustrated in (A) and (B), respectively, across three independent experiments. No statistical significant differences were found either in $(\mathbf{C})$ or (D).

Abbreviations: FITC, fluorescein isothiocyanate; NLC, nanostructured lipid carriers; TNF- $\alpha$, tumor necrosis factor $\alpha$; DC, dendritic cell.

\section{rsv-NLC modulate DC activity more efficiently than free rsv, and act by inhibiting NF- $\kappa \beta$ phosphorylation}

Having determined the concentrations of rsv that had to be encapsulated in NLC to be effective in inhibiting DC activation in response to TNF- $\alpha$, those were tested against the same concentrations of free rsv. Results are illustrated in Figure 7 and show a typical DC phenotypic profile in the different conditions. Also here, for all the conditions tested, the percentage of cells expressing the lineage markers (CD1a or CD11c) were maintained, indicating no dedifferentiation (Figure 7A). When results were quantified across different experiments, results indicate that, for the activation marker CD83, NLC encapsulating $5 \mu \mathrm{M}$ rsv behave very similarly to free $10 \mu \mathrm{M}$ rsv, although none of them showed a statistically significant difference from TNF- $\alpha$ alone. In this particular set of individuals, only NLC encapsulating $10 \mu \mathrm{M}$ rsv, but not free $10 \mu \mathrm{M}$ rsv, were shown to be able to significantly reduce CD83 expression (Figure 7B). Other phenotypic markers of DC activation were used to test if the effects observed 


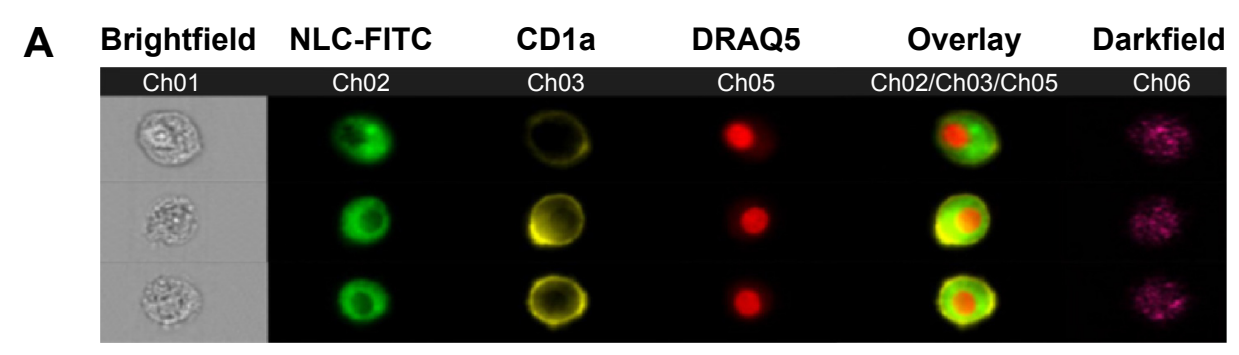

B NLC:

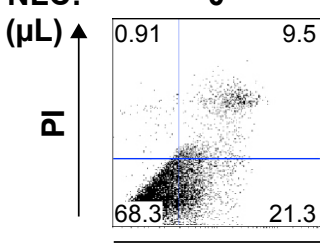

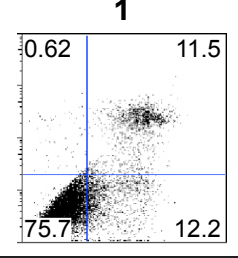
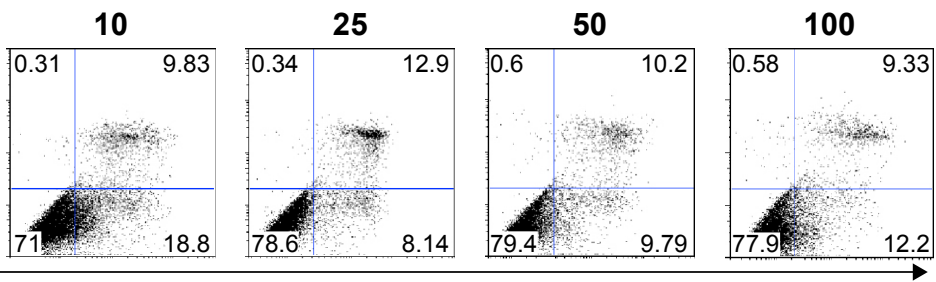

Annexin V

Figure 5 No cytotoxicity was observed upon NLC internalization.

Notes: (A) Representative images of DC morphology, upon NLC internalization, as analyzed by ImageStream ${ }^{\times}$. FITC (green)-loaded NLC were internalized by DC, which were then labeled for CDIa (yellow) and nuclei (DRAQ5, red). The overlay of the three channels is presented and cell complexity, in terms of intracellular vesicles, can be observed in the darkfield channel (purple). (B) Differentiated DCs were incubated with the indicated volumes of NLC for 24 hours, before being stained for Annexin $V$ and $\mathrm{Pl}$ and analyzed using a FACS Calibur flow cytometer.

Abbreviations: DC, dendritic cells; DRAQ5, deep red anthraquinone 5; FITC, fluorescein isothiocyanate; PI, propidium iodide; NLC, nanostructured lipid carriers.

were marker specific or instead related to particular classes of molecules. The results obtained indicate that, although there are no statistically significant differences, both major histocompatibility complex class I (HLA-ABC) and class II (HLA-DR) behave in a similar fashion (Figure 7B, compare top and bottom right graphs), while CD86 and CD40, two costimulatory molecules, also behave similarly (compare graphs in Figures 6B and 7B).

Phosphorylation levels of central transcription factor NF- $\kappa \beta$, known to be stimulated by TNF- $\alpha$, were probed in presence of rsv-NLC or free rsv. Results are illustrated in Figure 8A and show a marked reduction of NF- $\kappa \beta$ phosphorylation in the presence of rsv-NLC at 5 $\mu \mathrm{M}$, while free $10 \mu \mathrm{M}$ rsv only partially inhibited NF- $\kappa \beta$ phosphorylation, which was in agreement with our previous results. ${ }^{12}$

NF- $\kappa \beta$ activation targets the transcription of inflammatory cytokine genes, including the p40 subunit of IL-12/23. ${ }^{28}$ Thus, the levels of IL-12/23 p40 subunit, and anti-inflammatory cytokine IL-10, were quantified in the supernatant of DC cultures in the conditions described earlier (Figure 7), stimulated in presence or absence of rsv-NLC or free rsv, at 5 and $10 \mu \mathrm{M}$. Results illustrated in Figure 8B show that levels of IL-12/23 markedly increase in response to TNF- $\alpha$, as expected, and are significantly reduced when in presence of rsv-NLC 5 and $10 \mu \mathrm{M}$ or $10 \mu \mathrm{M}$ of free rsv (Figure 8B, top of graph). On the contrary, levels of IL-10 significantly decrease in the presence of TNF- $\alpha$, but do not appear to significantly increase in the presence of rsv-NLC or free rsv (Figure 8B, bottom of graph).

\section{Discussion}

Modulating DC activation is a desirable and hotly debated strategy, 2,29,30 both for promoting immunity as anticancer therapies or promoting tolerance for the control of autoimmune diseases such as rheumatoid arthritis. DCs are important players in immunity, including in the induction of central and peripheral tolerance, as demonstrated by mouse models, where the absence of DCs led to spontaneous autoimmunity. ${ }^{2}$

Delivery of different molecules to DCs is a current obstacle preventing the clinical translation of different therapeutic strategies. Different classes of nanoparticles, with or without specific molecular targeting, have been used previously to deliver molecules to DC. A recent work by Katakowski et al used liposomes decorated with DEC205 antibodies for targeting small interfering RNA (siRNA) to DCs, successfully knocking down their siRNA targets and inhibiting immune responses ${ }^{31}$ while the work by Warashina et al developed a polyethylene glycol-modified lipid nanoparticle also for siRNA delivery to DCs. ${ }^{32}$ In a previous work, Bhargava et al used solid lipid nanoparticles for delivering tumor antigens to DCs in order to promote antitumor immune responses, showing the advantages of manosylated versus nontargeted nanoparticles. ${ }^{33}$ Nonetheless, DC targeting nanoparticles for immune tolerance by targeting some of these receptors, 


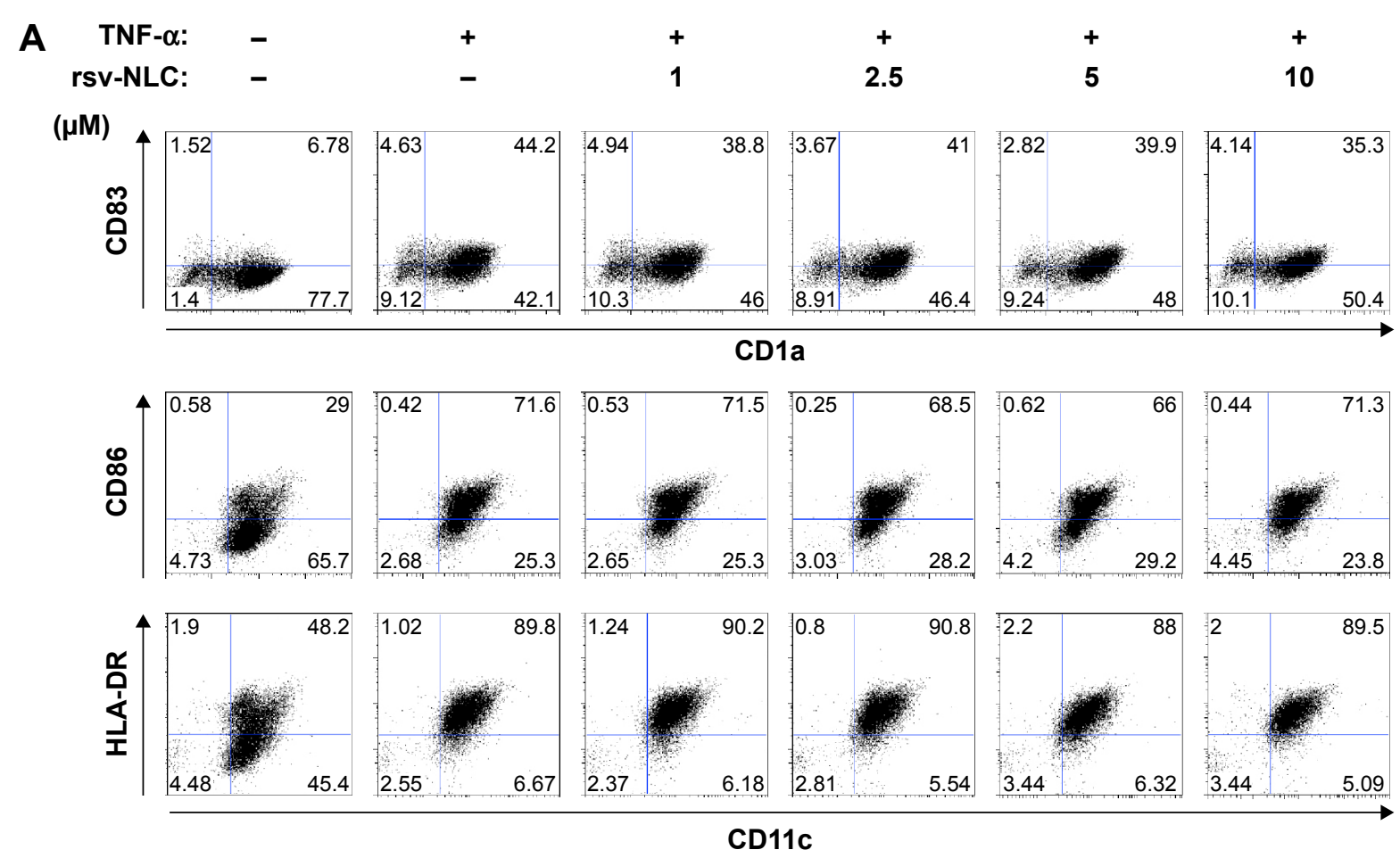

B

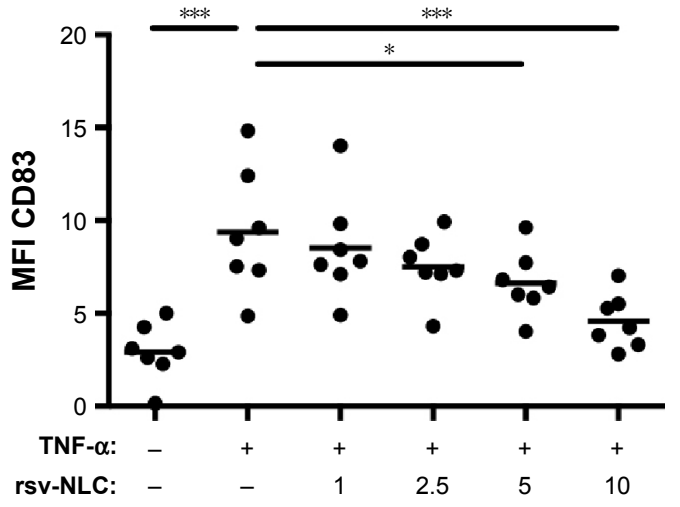
$(\mu \mathrm{M})$

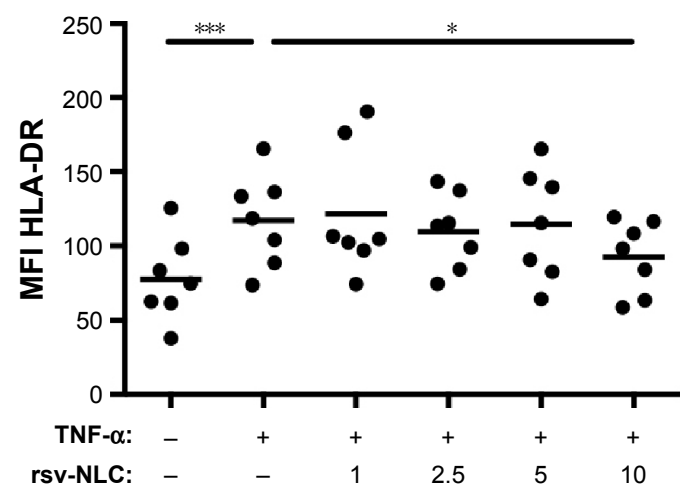
$(\mu \mathrm{M})$

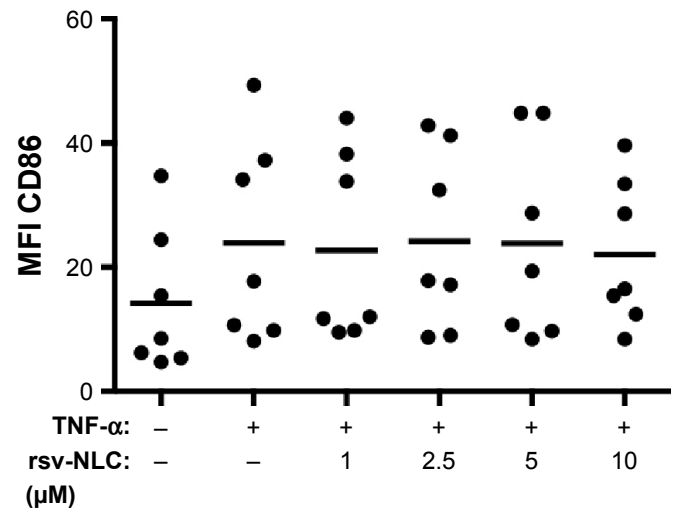

Figure 6 rsv-NLC reduce DC activation in response to TNF- $\alpha$.

Notes: Differentiated DCs were stimulated as indicated, with TNF- $\alpha$, in presence or absence of rsv-NLC for 72 h, before surface staining for CDIa and CD83 (top left), or CDIIc and CD86 (bottom), or HLA-DR (top right). Samples were analyzed using a FACS Calibur flow cytometer. (A) Representative plots of a phenotypic profile. (B) Graphs depict isotype control-subtracted MFI of CD83 (top left), HLA-DR (top right), or CD86 (bottom), over seven independent experiments. Each dot represents a different donor, and the line represents the mean value. Data were parametric and statistical significance was calculated using repeated measures analysis of variance, followed by Bonferroni's multiple comparison test $(* P<0.05$, ***P<0.00I).

Abbreviations: DC, dendritic cells; HLA-DR, human leukocyte antigen - antigen D related; MFI, mean fluorescence intensity; NLC, nanostructured lipid carriers; rsv, resveratrol; TNF- $\alpha$, tumor necrosis factor $\alpha$. 

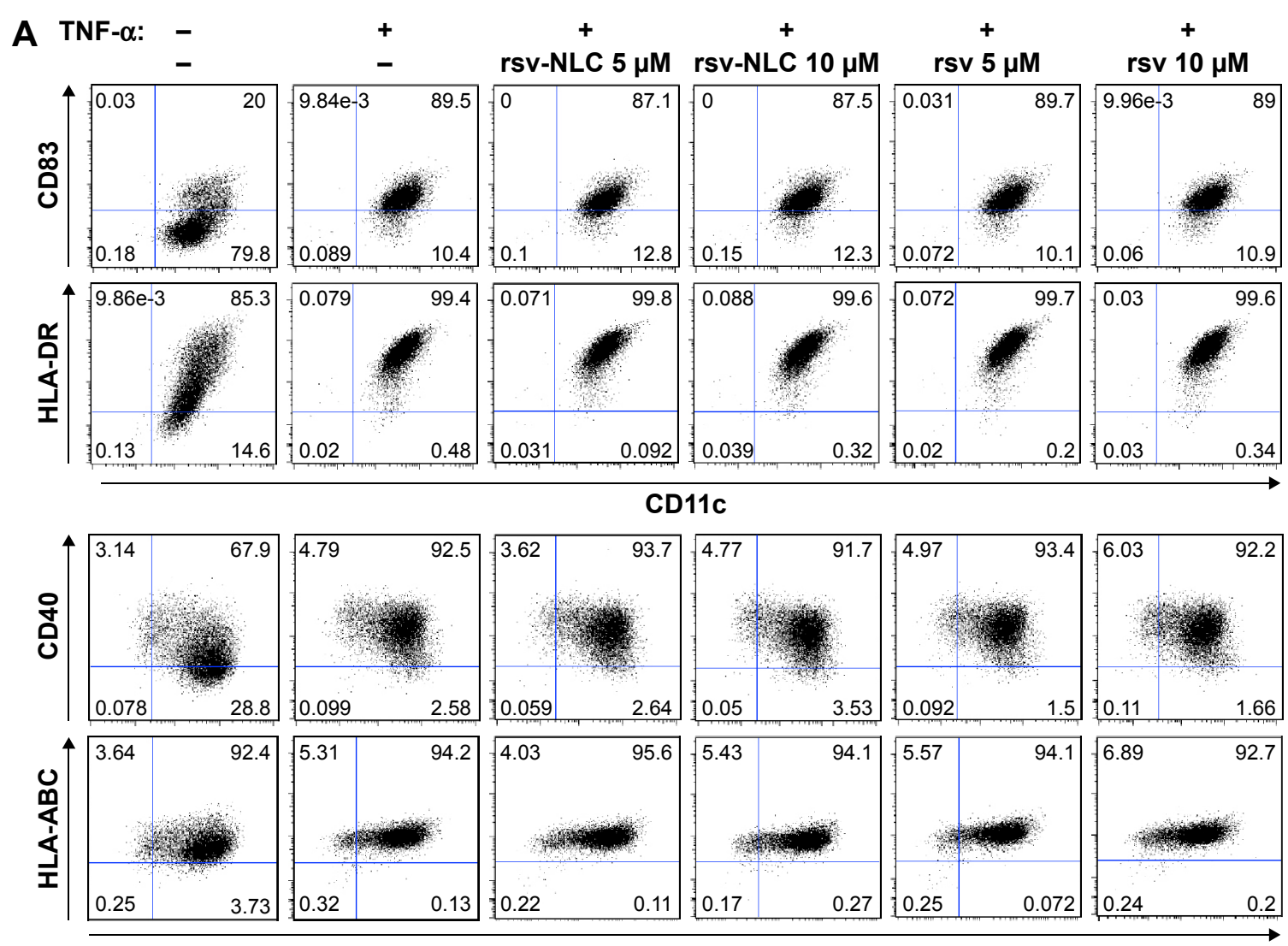

\section{CD1a}

B
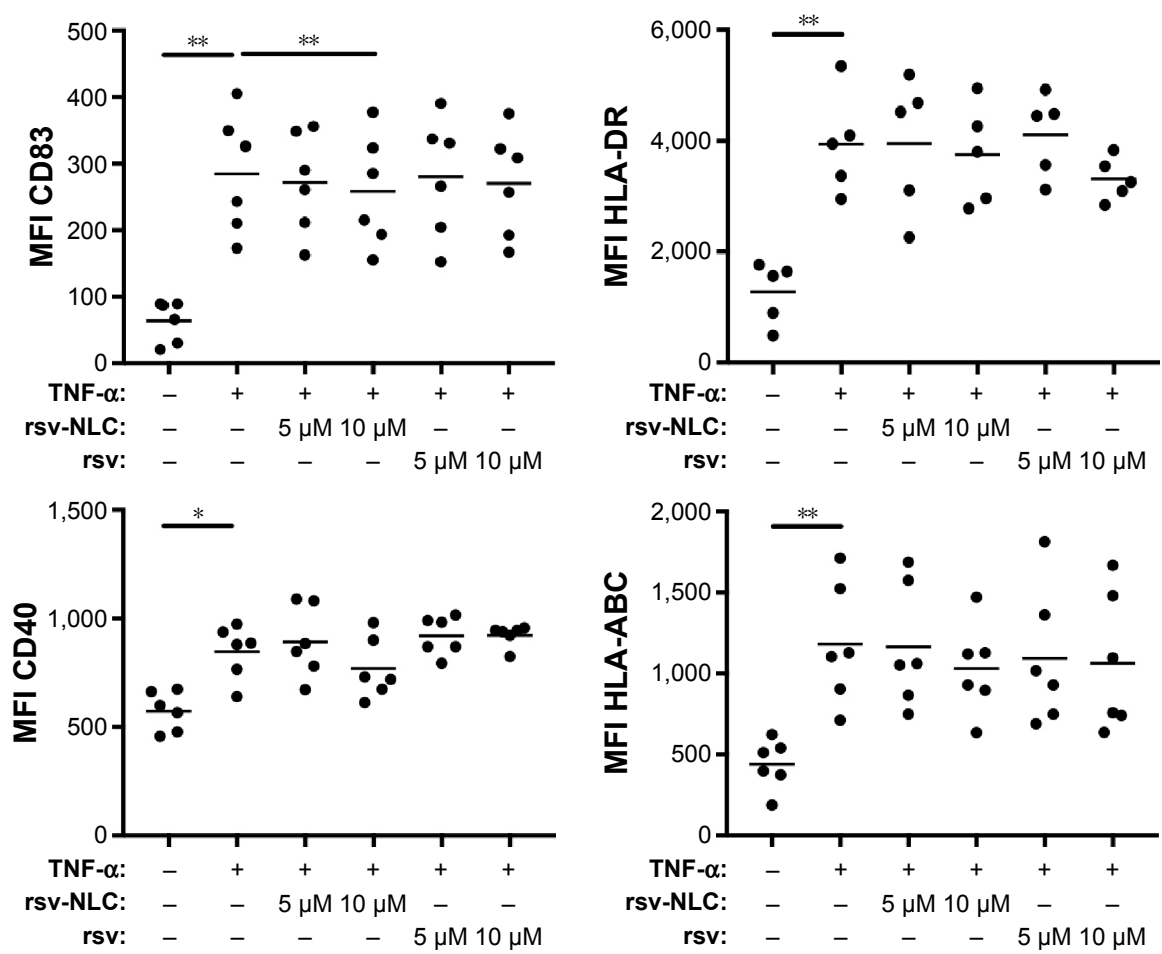

Figure 7 Effect of free versus NLC-encapsulated rsv on DC phenotype.

Notes: Differentiated DCs were stimulated as indicated, with TNF- $\alpha$, in the presence or absence of encapsulated rsv (rsv-NLC) or free rsv (rsv) for 72 hours, before surface staining for CD83 or HLA-DR and CDI Ic (top panel), and CD86 or HLA-ABC and CDIa (bottom panel). Samples were analyzed using a FACS Canto II flow cytometer. (A) Representative plots of a phenotypic profile. (B) Graphs depict isotype control-subtracted MFI of CD83 (top left), HLA-DR (top right), CD40 (bottom left), and HLA$A B C$ (bottom right) over six independent experiments. Each dot represents a different donor, and the line represents the mean value. Data were parametric and statistical significance was calculated using repeated measures analysis of variance, followed by Bonferroni's multiple comparison test ( $* P<0.05$, $* * P<0.0 \mathrm{I}$ ).

Abbreviations: DC, dendritic cells; HLA-ABC, human leukocyte antigen - antigens A, B, and C; HLA-DR, human leukocyte antigen - antigen D related; MFI, mean fluorescence intensity; NLC, nanostructured lipid carriers; rsv, resveratrol; TNF- $\alpha$, tumor necrosis factor $\alpha$. 
A

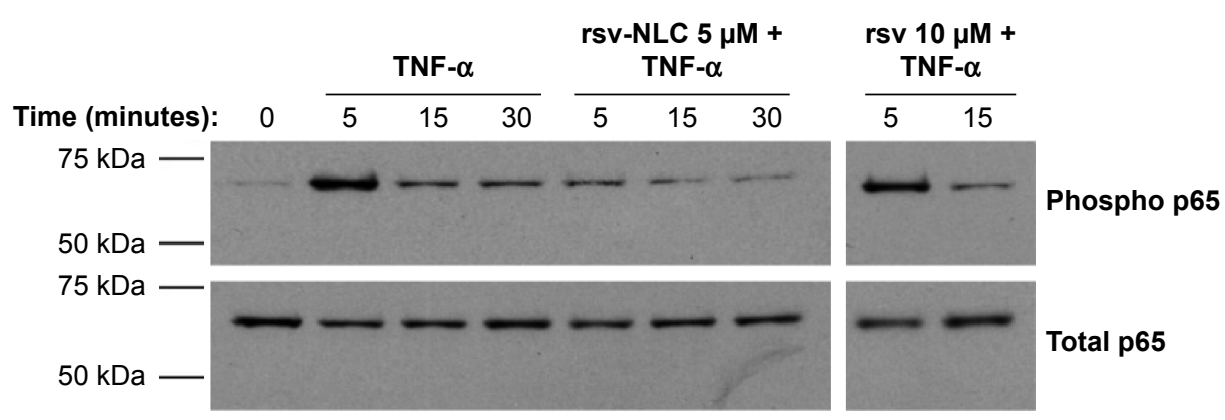

B
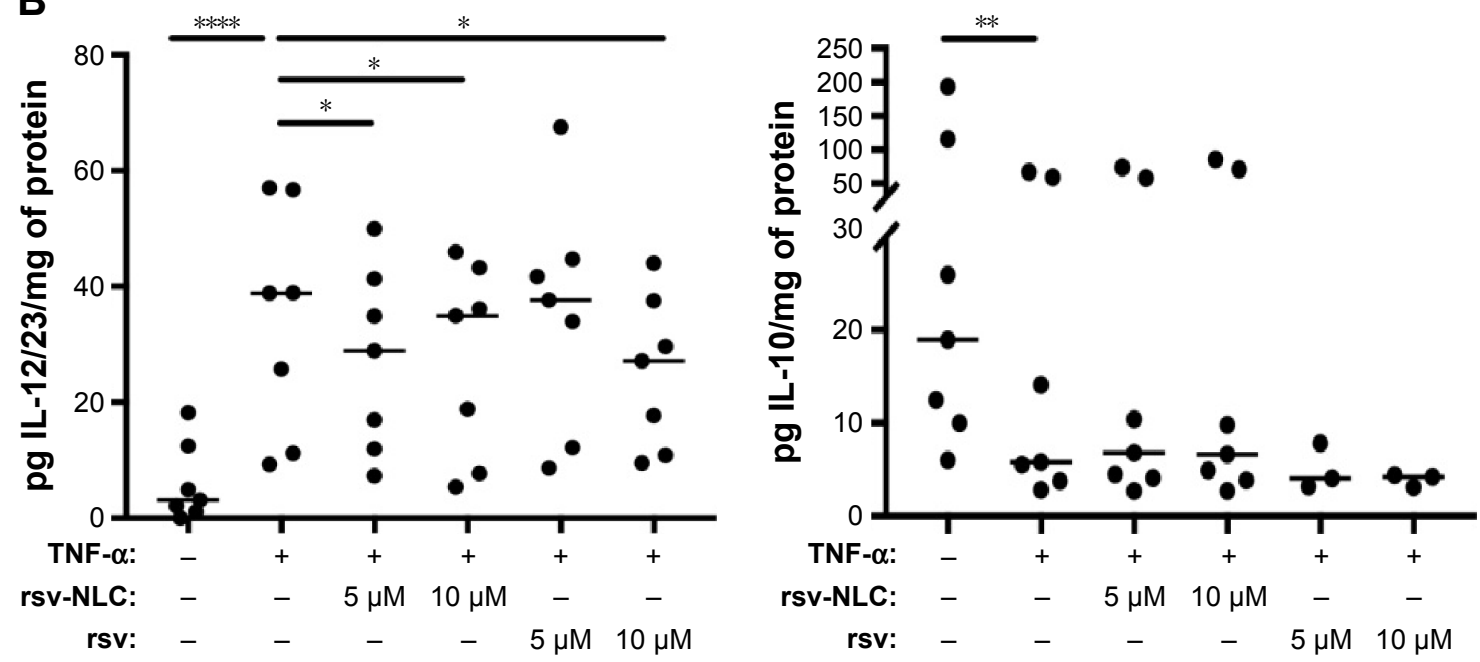

Figure 8 rsv-NLC affect NF- $\kappa \beta$ phosphorylation and cytokine secretion.

Notes: (A) Differentiated DCs were stimulated for the indicated times with TNF- $\alpha$, in presence or absence of rsv-NLC or free rsv. Cells were then harvested, lysed, and phosphorylation of $\mathrm{p} 65 \mathrm{NF}-\kappa \beta$ subunit (phosphor p65) analyzed by Western blot. Total $\mathrm{p} 65$ was also analyzed as control. (B) Differentiated DC were stimulated as indicated, with TNF- $\alpha$, in presence or absence of encapsulated rsv (rsv-NLC) or free rsv (rsv) for $72 \mathrm{~h}$. Supernatants were collected and cytokines IL-I2/23 and IL-I0 were measured by enzyme-linked immunosorbent assay. Each dot represents a different experiment, and the line represents the median value. Data were not parametric, so statistical significance was calculated using Friedman, followed by Dunn's uncorrected multiple comparison test $(* P<0.05, * * P<0.01, * * * * P<0.000 \mathrm{I})$.

Abbreviations: IL, interleukin; NLC, nanostructured lipid carriers; rsv, resveratrol; TNF- $\alpha$, tumor necrosis factor $\alpha$; DC, dendritic cell.

such as CD40, CD11c, or DEC205, has to be examined with care, as it may induce strong DC activation with IL-12 production and T-cell stimulation. ${ }^{34}$ Moreover, the route of administration and size of the nanoparticles also conditions the cells that will be targeted and the downstream outcome, for example, intravenously administered nanoparticles will likely be taken up by macrophages. ${ }^{34}$

For delivery of lipophilic compounds such as rsv, the use of NLC brings some advantages in terms of capacity of encapsulation and also for a more controlled release profile, due to the presence of small cavities in the lipid matrix that accommodate the compound. In comparison to liposomes, NLC require less time and are less expensive to prepare, and also do not require organic solvents for preparation. ${ }^{17}$ Here, NLC were produced combining high shear homogenization to produce particles in the micrometer range, with the ultrasound method used to reduce the microparticles to the nanometer range. The result was homogeneous FITC and rsv-NLC formulations with parameters that indicate stability, in agreement with what was previously described in the literature. ${ }^{19,35,36}$

In this study, the innovative imaging flow cytometry $\left(\right.$ ImageStream ${ }^{\mathrm{X}}$ ) technique was used to determine the percentage internalization of NLC by DCs. While conventional flow cytometry does not allow to distinguish nanoparticles associated with the cell membrane from truly internalized nanoparticles, ${ }^{37}$ in this study, by performing cell surface staining with a molecule that can function as a lineage marker (CD1a) and using the internalization algorithm, we were able to quantify only cells with internalized nanoparticles, and the images acquired allowed a high throughput confirmation of this result (Figure 3). Moreover, in relation to microscopy, which can determine true internalization with excellent spatial resolution, imaging flow cytometry allows an unbiased (not dependent on the operator) quantification, as gates are defined and then applied to all samples, on thousands of cells. ${ }^{37}$ Using this methodology, the dose-dependent NLC internalization and the effect of TNF- $\alpha$ stimulation 
on internalization were very clearly shown. The results observed cannot be attributed to cell stress and apoptosis, as the concentrations of NLC used in this study did not result in alterations in cell morphology or in the percentage of dead or apoptotic cells (Figure 5). Thus, FITC-NLC was shown to be suitable for labeling of DCs, without inducing significant changes in morphology, and imaging flow cytometry was revealed to be an effective technique to detect specific intracellular labeling.

Previous studies have shown the activity of rsv to counteract proinflammatory stimuli in a variety of cells. ${ }^{4-8}$ Moreover, it has been demonstrated to be effective for preventing DC activation in response to different stimuli. ${ }^{10-12,38}$ rsv has been reported as effective in ameliorating arthritis symptoms in animal models, ${ }^{39}$ and DCs are privileged targets to induce peripheral tolerance in arthritis. ${ }^{2}$ However, in most studies, rsv was added directly to culture media, leading to high doses being necessary for significant reduction of activation, for example, $50 \mu \mathrm{M}$ of rsv were effective in reducing DC stimulation by advanced glycation end products or lipopolysaccharide. ${ }^{11,38}$ Previous results from our team showed rsv efficacy in reducing DC response to TNF- $\alpha$, with $10 \mu \mathrm{M}$ of rsv being the necessary dose for a significant reduction of DC stimulation, as measured by CD83 expression. ${ }^{12}$ Yet, high doses have several drawbacks, including increased toxicity and cost, raising the importance of reducing working dosage to a minimum, by increasing bioavailability. In the current study, by encapsulating rsv into NLC, we were able to induce significant reduction of CD83 cell surface expression using only $5 \mu \mathrm{M}$ of rsv. Nonetheless, when free and encapsulated rsv were compared in the same set of individuals, only rsv-NLC, but not free rsv, at $10 \mu \mathrm{M}$ were able to significantly inhibit CD83 upregulation in response to TNF- $\alpha$. Furthermore, HLA-DR cell surface expression, which was significantly decreased in presence of NLC-encapsulated rsv, was then not confirmed when free versus NLC-encapsulated rsv were tested in parallel. On the other hand, the expression of costimulatory molecules, CD86 and CD40, do not vary significantly in the presence of NLC-encapsulated rsv, which differs from our previous results of CD86 expression in response to TNF- $\alpha$, and in the presence of free rsv. ${ }^{12}$ This is likely due to a mild stimulatory effect of the NLC, combined with the anti-inflammatory effect of rsv.

Moreover, in line with our previous results, ${ }^{12}$ the presence of either free rsv or rsv-NLC did not lead to reduced DC differentiation or detectable dedifferentiation, as determined by cell surface expression of lineage markers CD1a and CD11c, which remained relatively constant in all conditions tested. These results are different from those previously reported by other authors, where reduction of lineage markers was observed in the presence of free rsv. ${ }^{11}$

Functional consequences of exposure to rsv-NLC when stimulating with TNF- $\alpha$ were also evaluated. NF- $\kappa \mathrm{B}$ has been previously reported as a target for rsv in different cell lines ${ }^{15}$ and DCs. ${ }^{11} \mathrm{NF}-\kappa \mathrm{B}$ p65 phosphorylation is usually followed by translocation to the cell nucleus and activating transcription of target genes, including those of proinflammatory cytokines, such as the p40 subunit of IL-12 and IL-23. ${ }^{28}$ Our results show that NLC-encapsulated rsv at $5 \mu \mathrm{M}$ was more effective at reducing p 65 phosphorylation in response to TNF- $\alpha$ than free rsv at $10 \mu \mathrm{M}$ (Figure $8 \mathrm{~A}$ ), which led to only a partial inhibition of phosphorylation, in line with results previously reported by us. ${ }^{12}$ This correlated with statistically significant reductions in the levels of IL-12/23 in presence of rsv-NLC at 5 or $10 \mu \mathrm{M}$ and also free rsv at $10 \mu \mathrm{M}$, which is also in line with our previous results. ${ }^{12}$ The same was not verified for IL-10 secretion (Figure 8B). These functional effects of NLC-encapsulated rsv further confirm that NLC are capable of delivering functional rsv to DCs.

Although the results obtained show that NLC-encapsulated rsv remains stable and active in vitro, the in vivo capacity of these systems to increase rsv bioavailability remains to be fully demonstrated, as is their capacity to ameliorate inflammatory conditions. These would constitute important steps toward validation of their use in the clinical setting.

\section{Conclusion}

In conclusion, this study shows that NLC can be internalized by immature and mature DCs alike, rapidly and without inducing dedifferentiation or cytotoxicity. rsv encapsulated in NLC maintains its bioactivity, modulating the mechanisms of DC activation in response to a proinflammatory environment, even at very low concentrations. Thus, the current work clearly demonstrates the potential for NLC to deliver different molecules or dyes to human primary DCs.

\section{Acknowledgments}

The authors would like to thank the Serviço de Imunohemoterapia of Centro Hospitalar de São João, for kindly donating buffy coats and b.IMAGE - the Bioimaging Center for Biomaterials and Regenerative Therapies. This work was financed by UP and Santander (PP_IJUP2011_220); and FEDER - Fundo Europeu de Desenvolvimento Regional funds through the COMPETE 2020 - Operacional Programme for Competitiveness and Internationalisation (POCI), Portugal 2020, and by Portuguese funds through 
FCT - Fundação para a Ciência e a Tecnologia/Ministério da Ciência, Tecnologia e Inovação in the framework of the project "Institute for Research and Innovation in Health Sciences" (POCI-01-0145-FEDER-007274).

\section{Disclosure}

ARN and AMS were funded by $\mathrm{PhD}$ fellowships from FCTPOPH (SFRH/BD/73379/2010 and SFRH/BD/85968/2012).

The authors declare no other conflicts of interest in this work.

\section{References}

1. Seth A, Oh DB, Lim YT. Nanomaterials for enhanced immunity as an innovative paradigm in nanomedicine. Nanomedicine (Lond). 2015; 10(6):959-975.

2. Schinnerling K, Soto L, Garcia-Gonzalez P, Catalan D, Aguillon JC. Skewing dendritic cell differentiation towards a tolerogenic state for recovery of tolerance in rheumatoid arthritis. Autoimmun Rev. 2015; 14(6):517-527.

3. Neves AR, Lucio M, Lima JL, Reis S. Resveratrol in medicinal chemistry: a critical review of its pharmacokinetics, drug-delivery, and membrane interactions. Curr Med Chem. 2012;19(11):1663-1681.

4. Ahn J, Lee H, Kim S, Ha T. Resveratrol inhibits TNF-alpha-induced changes of adipokines in 3T3-L1 adipocytes. Biochem Biophys Res Commun. 2007;364(4):972-977.

5. Bertelli AA, Baccalini R, Battaglia E, Falchi M, Ferrero ME. Resveratrol inhibits TNF alpha-induced endothelial cell activation. Therapie. 2001;56(5):613-616.

6. Bi XL, Yang JY, Dong YX, et al. Resveratrol inhibits nitric oxide and TNF-alpha production by lipopolysaccharide-activated microglia. Int Immunopharmacol. 2005;5(1):185-193.

7. Buttari B, Profumo E, Segoni L, et al. Resveratrol counteracts inflammation in human M1 and M2 macrophages upon challenge with 7-oxo-cholesterol: potential therapeutic implications in atherosclerosis. Oxid Med Cell Longev. 2014;2014:257543.

8. Csaki C, Keshishzadeh N, Fischer K, Shakibaei M. Regulation of inflammation signalling by resveratrol in human chondrocytes in vitro. Biochem Pharmacol. 2008;75(3):677-687.

9. Elmali N, Esenkaya I, Harma A, Ertem K, Turkoz Y, Mizrak B. Effect of resveratrol in experimental osteoarthritis in rabbits. Inflamm Res. 2005;54(4):158-162.

10. Kim GY, Cho H, Ahn SC, Oh YH, Lee CM, Park YM. Resveratrol inhibits phenotypic and functional maturation of murine bone marrowderived dendritic cells. Int Immunopharmacol. 2004;4(2):245-253.

11. Svajger U, Obermajer N, Jeras M. Dendritic cells treated with resveratrol during differentiation from monocytes gain substantial tolerogenic properties upon activation. Immunology. 2010;129(4):525-535.

12. Silva AM, Oliveira MI, Sette L, et al. Resveratrol as a natural antitumor necrosis factor-alpha molecule: implications to dendritic cells and their crosstalk with mesenchymal stromal cells. PLoS One. 2014;9(3):e91406.

13. Csiszar A, Smith K, Labinskyy N, Orosz Z, Rivera A, Ungvari Z. Resveratrol attenuates TNF-alpha-induced activation of coronary arterial endothelial cells: role of NF-kappaB inhibition. Am J Physiol Heart Circ Physiol. 2006;291(4):H1694-H1699.

14. Kang OH, Jang HJ, Chae HS, et al. Anti-inflammatory mechanisms of resveratrol in activated HMC-1 cells: pivotal roles of NF-kappaB and MAPK. Pharmacol Res. 2009;59(5):330-337.

15. Manna SK, Mukhopadhyay A, Aggarwal BB. Resveratrol suppresses TNF-induced activation of nuclear transcription factors NF-kappa B, activator protein-1, and apoptosis: potential role of reactive oxygen intermediates and lipid peroxidation. J Immunol. 2000;164(12):6509-6519.
16. Wang $\mathrm{X}$, Zhang C, Huang G, et al. Resveratrol inhibits dysfunction of dendritic cells from chronic obstructive pulmonary disease patients through promoting miR-34. Int J Clin Exp Pathol. 2015;8(5):5145-5152.

17. Summerlin N, Soo E, Thakur S, Qu Z, Jambhrunkar S, Popat A. Resveratrol nanoformulations: challenges and opportunities. Int J Pharm. 2015;479(2):282-290.

18. Lim SB, Banerjee A, Onyuksel H. Improvement of drug safety by the use of lipid-based nanocarriers. J Control Release. 2012;163(1):34-45.

19. Müller RH, Mäder K, Gohla S. Solid lipid nanoparticles (SLN) for controlled drug delivery - a review of the state of the art. Eur J Pharm Biopharm. 2000;50(1):161-177.

20. Neves AR, Lucio M, Martins S, Lima JL, Reis S. Novel resveratrol nanodelivery systems based on lipid nanoparticles to enhance its oral bioavailability. Int J Nanomedicine. 2013;8:177-187.

21. Gokce EH, Korkmaz E, Dellera E, Sandri G, Bonferoni MC, Ozer O. Resveratrol-loaded solid lipid nanoparticles versus nanostructured lipid carriers: evaluation of antioxidant potential for dermal applications. Int J Nanomedicine. 2012;7:1841-1850.

22. Selvamuthukumar S, Velmurugan R. Nanostructured lipid carriers: a potential drug carrier for cancer chemotherapy. Lipids Health Dis. 2012;11:159.

23. Basha G, Novobrantseva TI, Rosin N, et al. Influence of cationic lipid composition on gene silencing properties of lipid nanoparticle formulations of siRNA in antigen-presenting cells. Mol Ther. 2011; 19(12):2186-2200.

24. Erni C, Suard C, Freitas S, Dreher D, Merkle HP, Walter E. Evaluation of cationic solid lipid microparticles as synthetic carriers for the targeted delivery of macromolecules to phagocytic antigen-presenting cells. Biomaterials. 2002;23(23):4667-4676.

25. Oliveira MI, Santos SG, Oliveira MJ, Torres AL, Barbosa MA. Chitosan drives anti-inflammatory macrophage polarisation and pro-inflammatory dendritic cell stimulation. Eur Cell Mater. 2012;24:136-152; discussion 152-153.

26. Sallusto F, Lanzavecchia A. Efficient presentation of soluble antigen by cultured human dendritic cells is maintained by granulocyte/macrophage colony-stimulating factor plus interleukin 4 and downregulated by tumor necrosis factor alpha. J Exp Med. 1994;179(4):1109-1118.

27. Chapuis F, Rosenzwajg M, Yagello M, Ekman M, Biberfeld P, Gluckman JC. Differentiation of human dendritic cells from monocytes in vitro. Eur J Immunol. 1997;27(2):431-441.

28. Pahl HL. Activators and target genes of Rel/NF-kappaB transcription factors. Oncogene. 1999;18(49):6853-6866.

29. Garg AD, Romano E, Rufo N, Agostinis P. Immunogenic versus tolerogenic phagocytosis during anticancer therapy: mechanisms and clinical translation. Cell Death Differ. 2016;23(6):938-951.

30. Chen K, Wang JM, Yuan R, et al. Tissue-resident dendritic cells and diseases involving dendritic cell malfunction. Int Immunopharmacol. 2016;34:1-15.

31. Katakowski JA, Mukherjee G, Wilner SE, et al. Delivery of siRNAs to dendritic cells using DEC205-targeted lipid nanoparticles to inhibit immune responses. Mol Ther. 2016;24(1):146-155.

32. Warashina S, Nakamura T, Sato Y, et al. A lipid nanoparticle for the efficient delivery of siRNA to dendritic cells. J Control Release. 2016;225:183-191.

33. Bhargava A, Mishra D, Khan S, Varshney SK, Banerjee S, Mishra PK. Assessment of tumor antigen-loaded solid lipid nanoparticles as an efficient delivery system for dendritic cell engineering. Nanomedicine (Lond). 2013;8(7):1067-1084.

34. Getts DR, Shea LD, Miller SD, King NJ. Harnessing nanoparticles for immune modulation. Trends Immunol. 2015;36(7):419-427.

35. Muller RH, Schwarz C, Mehnert W, Lucks JS. Production of solid lipid nanoparticles (SLN) for controlled drug delivery. Proc Int Symp Control Release Bioact Mater. 1993;20:480-481.

36. Jenning V, Gohla S, Muller RH. Solid lipid nanoparticles (SLN): effect of homogenization parameters on drug stability. In: Proceedings 2nd World Meeting APGI/APV; Paris; 1998:619-620. 
37. Phanse Y, Ramer-Tait AE, Friend SL, et al. Analyzing cellular internalization of nanoparticles and bacteria by multi-spectral imaging flow cytometry. J Vis Exp. 2012;(64):e3884.

38. Buttari B, Profumo E, Facchiano F, et al. Resveratrol prevents dendritic cell maturation in response to advanced glycation end products. Oxid Med Cell Longev. 2013;2013:574029.
39. Elmali N, Baysal O, Harma A, Esenkaya I, Mizrak B. Effects of resveratrol in inflammatory arthritis. Inflammation. 2007;30(1-2):1-6. 


\section{Supplementary materials}

Nanostructured lipid carriers (NLC) formulations

The lipid components of the future emulsion for fluorescein isothiocyanate (FITC) and resveratrol (rsv)-loaded NLC are illustrated in Table S1.

\section{NLC production and characterization}

rsv EE was calculated along time, showing that time in storage did not influence rsv EE (Figure S2).

\section{FITC-NLC internalization by DC as analyzed by imaging flow cytometry (ImageStream $\times$ )}

The workflow used during the IDEAS' "Internalization" wizard is illustrated in Figure S1. To ensure that the events (or cells) under analysis were in focus, a histogram according to the gradient RMS (enables the discrimination between focused and unfocused cells, by enumerating changes of pixel values) was created, and a gate was applied from the minimal value that presented cells in focus (population named "Focus"). Then, for the events of "Focus", a dot plot of their area was made in order to select only the events that corresponded to single cells (population named "Focus \& Single"). After that, cell events from "Focus \& Single" were chosen according to the CD1a fluorescence intensity (blood monocyte-derived DC express CD1a de novo), defining the CD1a positive DC population (population named "Focus \& Single \& CD1a"). The gated events were then displayed in a dot plot for the intensity of the fluorescence acquired in channel 02 (Ch02), where FITC-NLC should be visible, with the maximum fluorescence values (Max Pixel Ch02). Taking this into account, a gate for cells showing NLC internalization was defined (named R1).

Table SI Preparation of FITC and rsv-loaded NLC

\begin{tabular}{lllllll}
\hline NP Formulation & FITC $(\mathbf{m g})$ & rsv $(\mathbf{m g})$ & Cetyl palmitate $(\mathbf{m g})$ & Miglyol-8 I 2 (mg) & Tween 60 (mg) & Water (mL) \\
\hline Nonloaded NLC (nl-NLC) & - & - & 350 & 150 & 100 & 4.4 \\
2 mg FITC-loaded NLC (FITC2-NLC) & 2 & - & 348 & 150 & 100 & 4.4 \\
5 mg FITC-loaded NLC (FITC5-NLC) & 5 & - & 345 & 150 & 100 & 4.4 \\
8 mg RSV-loaded NLC (RSV8-NLC) & - & 8 & 342 & 150 & 100 & 4.4 \\
\hline
\end{tabular}

Abbreviations: FITC, fluorescein isothiocyanate; NLC, nanostructured lipid carriers; rsv, resveratrol.
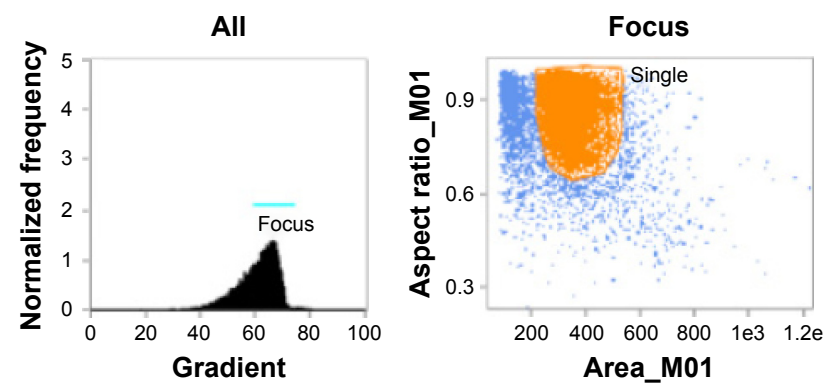

RMS_M01_Ch01
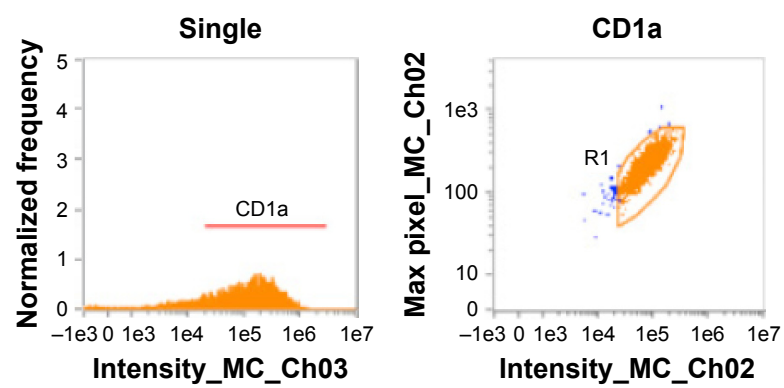

Figure SI Workflow for determination of percentage of FITC-NLC internalization.

Notes: Gates define population in focus (top left), excluding cell duplets or other complexes (top right), defining CD la positive cells (bottom left) and FITC intensity (bottom right). Gate RI shows positive events for NLC internalization.

Abbreviations: FITC, fluorescein isothiocyanate; NLC, nanostructured lipid carriers. 


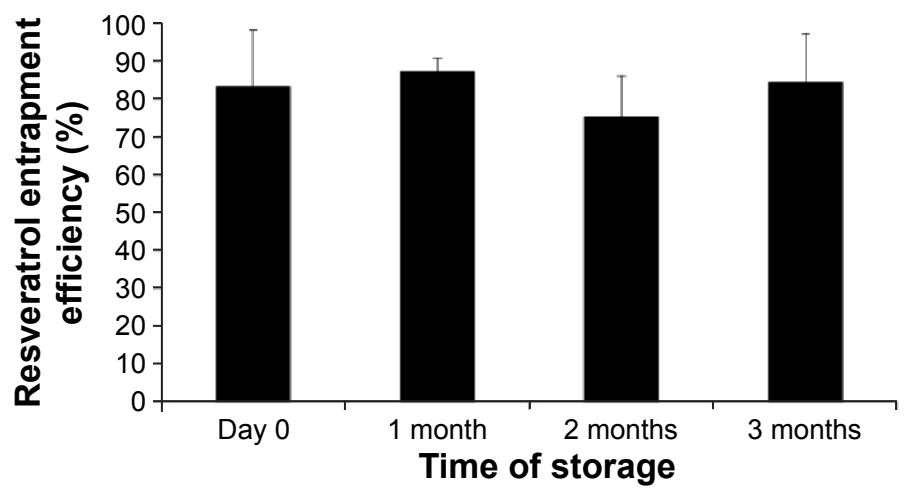

Figure S2 Effect of time of storage on resveratrol entrapment efficiency of NLC.

Notes: The analysis was performed after production (day 0 ) and along 3 months of storage. All data represent the mean \pm SD ( $n=3$ ). No statistically significant differences were found $(P>0.05)$.

Abbreviations: SD, standard deviation; NLC, nanostructured lipid carriers.

\section{Publish your work in this journal}

The International Journal of Nanomedicine is an international, peerreviewed journal focusing on the application of nanotechnology in diagnostics, therapeutics, and drug delivery systems throughout the biomedical field. This journal is indexed on PubMed Central, MedLine, CAS, SciSearch $®$, Current Contents ${ }^{\circledR} /$ Clinical Medicine,
Journal Citation Reports/Science Edition, EMBase, Scopus and the Elsevier Bibliographic databases. The manuscript management system is completely online and includes a very quick and fair peer-review system, which is all easy to use. Visit http://www.dovepress.com/ testimonials.php to read real quotes from published authors.

Submit your manuscript here: http://www.dovepress.com/international-journal-of-nanomedicine-journal 